\title{
ANATOMIA SISTEMÁTICA E ECOLÓGICA DA MADEIRA DE SEIS ESPÉCIES DE NECTANDRA ROL. EX ROTTB. (LAURACEAE) ${ }^{1}$
}

\author{
SUELIZE THOMAZ HEERDT ${ }^{2}$ JOÃO CARLOS FERREIRA DE MELO JÚNIOR ${ }^{3}$
}

\section{RESUMO}

A análise comparativa da madeira de seis espécies de Nectandra (Lauraceae) reforça a grande uniformidade estrutural atribuída ao gênero. Caracteres como tipo de placa de perfuração, presença de tilos, diâmetro de pontoações intervasculares, comprimento de fibras, altura de raios, bem como a presença de raios agregados e de células oleíferas/mucilaginosas podem contribuir para a segregação das espécies. As diferenças em caracteres quantitativos, por sua vez, podem ser respostas plásticas às condições ambientais dos ecossistemas de ocorrência das mesmas.

Palavras-chave: anatomia da madeira, anatomia sistemática, anatomia ecológica, Lauraceae, Nectandra.

\section{ABSTRACT}

[Taxonomic and ecological wood anatomy of six species of Nectandra Rol. ex Rottb. (Lauraceae)]. The comparative analysis of six wood species of Nectandra (Lauraceae) reinforces the great structural uniformity presented by genus. Characters such as type of perforation plates, tyloses, intervessel pit size, fiber size, ray height, aggregate rays and presence of oil/mucilaginous cells can contribute to the segregation of species. On the other hand, the quantitative differences between characters can be plastic responses to environmental conditions of occurrence of the species ecosystem.

Keywords: ecological anatomy, Lauraceae, Nectandra, systematic anatomy, wood anatomy.

\section{INTRODUÇÃO E REVISÃO DE LITERA- TURA}

Lauraceae Juss. é uma família de Angiospermas basais de distribuição predominantemente pantropical, que está bem representada na América, Ásia tropical, Austrália, Madagascar e, mais esparsamente, na África Central (Van Der Werff \& Richter, 1996). Algumas espécies são restritas a regiões subtropicais e poucas alcançam latitudes temperadas (Madriñán, 2004). Composta, aproximadamente, de 52 gêneros e 2.500 a 3.000 espécies (Rohwer,1993), apresenta 24 gêneros e 434 espécies no Brasil, as quais são geralmente arbóreas e habitam Florestas Pluviais, Restingas e Cerrados (Barroso, 2002), destacando-se na composição florística

1 Recebido em 09-6-2016 e aceito para publicação em 25-7-2016.

2 Acadêmica, Curso de Ciências Biológicas - Meio Ambiente e Biodiversidade, Departamento de Ciências Biológicas, Universidade da Região de Joinville, Joinville, Santa Catarina, Brasil. suelize@ gmail.com

3 Biólogo, Doutor, Professor Titular do Departamento de Ciências Biológicas, Laboratório de Anatomia e Ecologia Vegetal, Universidade da Região de Joinville, Joinville, Santa Catarina, Brasil. joao.melo@univille.br de grande parte dos ecossistemas florestais do país, notadamente da Mata Atlântica (Lorenzi, 2012).

A família constitui um dos grupos taxonômicos mais complexos para a caracterização das espécies, em função de sua elevada diversidade e por serem utilizados caracteres crípticos na distinção de gêneros e espécies (Lorenzi, 2012). A acentuada uniformidade morfológica (Oliveira et al., 2001) e grande homogeneidade de caracteres anatômicos (Cronquist, 1988) dificultam a caracterização (Van Der Werff \& Richter, 1996), fato que explica a inexistência de uma classificação convincente, baseada, prioritariamente, na evidência morfológica, salientando-se, no caso da anatomia, o escasso conhecimento sobre a variabilidade estrutural do lenho na família como um todo (Metcalfe, 1987).

O gênero Nectandra Rol. ex Rottb., de elevada diversidade em número de espécies (Quinet \& Andreata, 2002), apresenta 43 espécies nativas no Brasil, distribuídas em todos os biomas (Quinet, 2015). Este gênero acompanha a alta complexidade taxonômica e uniformidade 
morfológica atribuída à família (Castiglioni, 1962); a maioria das chaves de identificação para a mesma baseia-se em caracteres florais (Van Der Werff, 1991), sendo ainda muito incipientes os dados moleculares (Chanderbali et al., 2001) e muito parecida a embriologia das espécies (Heo et al., 1998). Dados anatômicos sugerem certo grau de confiança e, por isso, são usados para resolver problemas taxonômicos e relações filogenéticas, além de ajudar a interpretar direções evolucionárias e ecológicas (Stuessy, 1991).

Com o presente estudo pretende-se contribuir para a ampliação de conhecimentos sobre a anatomia de espécies do gênero Nectandra, com especial atenção aos caracteres úteis para a identificação das mesmas e sua interpretação ecológica.

\section{MATERIAL E MÉTODOS}

O material botânico estudado é proveniente de três diferentes regiões fitogeográficas do Brasil: Floresta Estacional Semidecídua (FES), Floresta Ombrófila Densa de Terras Baixas (FODTB) e Restinga (Tabela 1) e encontra-se tombado na xiloteca JOIw (Melo Jr. et al., 2014). Seis espécies foram selecionadas para estudo, com base na disponibilidade de amostras da coleção: N. lanceolata Nees, N. membranacea (Sw) Griseb., N. oppositifolia Nees, $N$. psammophila Nees, N. puberula (Schott) Nees e N. reticulata (Ruiz \&Pav.) Mez.

TABELA 1. Dados climatológicos e pluviométricos dos locais de coleta das espécies de Nectandra Rol. ex Rottb. (Lauraceae).

\begin{tabular}{|c|c|c|c|c|c|c|c|}
\hline 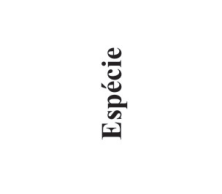 & 宽 & 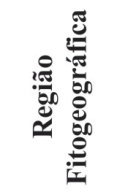 & 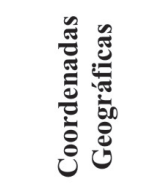 & 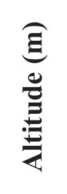 & 咅高 & 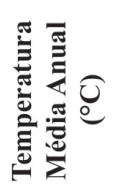 & 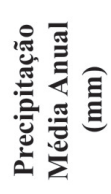 \\
\hline N. lanceolata & $\begin{array}{c}\text { Matozinhos } \\
\text { (MG) }\end{array}$ & FES & $\begin{array}{l}19^{\circ} 28^{\prime} 23,8^{\prime \prime} \\
43^{\circ} 59^{\prime} 15,2^{\prime \prime}\end{array}$ & 812 & Aw & 21,6 & 1321 \\
\hline N. membranacea & $\begin{array}{c}\text { São } \\
\text { Francisco do } \\
\text { Sul (SC) }\end{array}$ & FODTB & $\begin{array}{l}26^{\circ} 18^{\prime} 45,5^{\prime \prime} \\
48^{\circ} 38^{\prime} 39,1^{\prime \prime}\end{array}$ & 9 & $\mathrm{Cfa}$ & 21,2 & 1830 \\
\hline N. oppositifolia & $\begin{array}{c}\text { São } \\
\text { Francisco do } \\
\text { Sul }(\mathrm{SC})\end{array}$ & REST & $\begin{array}{l}26^{\circ} 16^{\prime} 58,1^{\prime \prime} \\
48^{\circ} 02^{\prime} 14,7^{\prime \prime}\end{array}$ & 18 & Cfa & 21,2 & 1830 \\
\hline N. psammophila & $\begin{array}{l}\text { Matozinhos } \\
\text { (MG) }\end{array}$ & FES & $\begin{array}{l}19^{\circ} 28^{\prime} 37,9^{\prime \prime} \\
44^{\circ} 00^{\prime} 54,6 "\end{array}$ & 812 & Aw & 21,6 & 1321 \\
\hline N. puberula & $\begin{array}{l}\text { Guaramirim } \\
\text { (SC) }\end{array}$ & FODTB & $\begin{array}{l}26^{\circ} 27^{\prime} 15,7^{\prime \prime} \\
48^{\circ} 55^{\prime} 45,6 "\end{array}$ & 23 & $\mathrm{Cfa}$ & 20,9 & 1613 \\
\hline N. reticulata & $\begin{array}{l}\text { Araquari } \\
\text { (SC) }\end{array}$ & FODTB & $\begin{array}{l}26^{\circ} 22^{\prime} 11,4^{\prime \prime} \\
48^{\circ} 40^{\prime} 48,4^{\prime \prime}\end{array}$ & 34 & Cfa & 21,1 & 1745 \\
\hline
\end{tabular}

Onde: FES, floresta estacional semidecidual; FODTB, floresta ombrófila densa de terras baixas; REST, restinga; MG, estado das Minas Gerais; SC, estado de Santa Catarina. As informações climáticas foram extraídas de Climate-Data (2016). 
Preparações histológicas foram realizadas de acordo com as técnicas usuais em anatomia da madeira (Johansen, 1940; Sass, 1958; Paiva et al., 2006). Macerações foram preparadas por meio da solução de Franklin, modificada por Kraus \& Arduin (1997), para posterior biometria de vasos (comprimento e diâmetro tangencial) e fibras (espessura da parede), $\operatorname{com} n=25$. A densidade básica da madeira foi obtida pelo método da balança hidrostática (ABNT, 2002). As microfotografias foram capturadas com fotomicroscópio Olympus CX-31. As mensurações foram feitas por meio do software Dino Eye 2.0.

As descrições anatômicas seguiram a proposta da IAWA Committee (1989). Para todos os atributos quantitativos da madeira foram calculadas as médias e os respectivos desvios-padrão. Para o teste de normalidade de resíduos, aplicou-se ANOVA, post hoc Tukey, com $\mathrm{p}<0,05$, para comparar as médias dos atributos entre as espécies estudadas em ambiente $\mathrm{R}$ (Borcard et al., 2011).

\section{RESULTADOS}

As espécies de madeira em estudo são anatomicamente descritas a seguir, por ordem alfabética, sendo os dados quantitativos apresentados na Tabela 2. A Figura 1 ilustra as estruturas descritas.

\section{Nectandra lanceolata Nees}

Anéis de crescimento distintos, marcados por fibras de lenho tardio de paredes espessas. Vasos difusos, em múltiplos radiais 2-3 e solitários; diâmetro tangencial 100-200 $\mu \mathrm{m}$; frequência 20-40 por $\mathrm{mm}^{2}$; placas de perfuração simples e escalariformes com poucas barras; pontoações intervasculares alternas, pequenas $(4-7 \mu \mathrm{m})$. Pontoações radio-vasculares simples, com borda reduzida, horizontal. Fibras com pontoações simples e aréolas reduzidas em vista radial; septos presentes; paredes finas. Parênquima axial paratraqueal escasso e vasicêntrico; série fusiforme de parênquima axial com 2-3 células. Raios (1-3) 2-3 seriados, com 6-78 células de altura; heterocelulares, com corpo de células procumbentes e margens de células eretas ou quadradas.

\section{Nectandra membranacea (Sw.) Griseb.}

Anéis de crescimento distintos, marcados por fibras de lenho tardio com paredes espessas. Vasos difusos, solitários e em múltiplos de dois; diâmetro tangencial $=200 \mu \mathrm{m}$; frequência 5-20 por $\mathrm{mm}^{2}$; placas de perfuração simples e foraminadas; pontoações intervasculares alternas, grandes $(>10 \mu \mathrm{m})$; pontoações radiovasculares simples, com borda reduzida, horizontal. Fibras de paredes finas, com pontoações simples e aréolas reduzidas em vista radial; septos, presentes. Parênquima axial paratraqueal escasso; séries fusiformes com 3-5 células. Raios (1-3) 2-3 seriados, com 7-40 células de altura; heterocelulares, com corpo de células quadradas e procumbentes e margens de células eretas.

\section{Nectandra oppositifolia Nees}

Anéis de crescimento distintos, marcados por fibras de lenho tardio com paredes espessas. Vasos difusos, solitários e em múltiplos de dois; diâmetro tangencial 100-200 ㅆm; frequência 520 por $\mathrm{mm}^{2}$; placas de perfuração simples e escalariformes de poucas barras; pontoações intervasculares alternas, pequenas $(4-7 \mu \mathrm{m})$; pontoações radio-vasculares simples, com borda reduzida, horizontal. Fibras de paredes finas, com pontoações simples e aréolas reduzidas em vista radial; septos, presentes. Parênquima axial paratraqueal escasso e vasicêntrico confluente com trecho curto; séries de parênquima axial > 8 células. Raios (1-3) 2-seriados, com 4-33 células de altura; heterocelulares, com corpo de células procumbentes e margens de células eretas ou quadradas.

\section{Nectandra psammophila Nees}

Anéis de crescimento distintos, marcados por fibras de lenho tardio de paredes espessas. Vasos difusos, solitários e em múltiplos de dois;

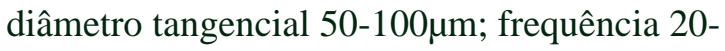


40 por $\mathrm{mm}^{2}$; placas de perfuração simples e escalariformes de poucas barras; pontoações intervasculares alternas, pequenas $(4-7 \mu \mathrm{m})$; pontoações radio-vasculares simples, com borda reduzida, horizontal. Fibras de paredes finas, com pontoações simples e aréolas reduzidas em vista radial; septos presentes. Parênquima axial paratraqueal escasso; séries de parênquima axial com 5-9 células. Raios (1-3) 2-3 seriados, com 5-70 células de altura; heterocelulares, com corpo de células procumbentes e margens de células eretas ou quadradas. Células oleíferas ou mucilaginosas, associadas aos parênquimas radial e axial.

\section{Nectandra puberula (Schott) Nees}

Anéis de crescimento distintos, marcados por fibras de lenho tardio de paredes espessas. Vasos difusos, em múltiplos de 2-3 e solitários; diâmetro tangencial 50-100 $\mu \mathrm{m}$; frequência 2040 por $\mathrm{mm}^{2}$; placas de perfuração simples e escalariformes de poucas barras; pontoações intervasculares alternas, pequenas $(4-7 \mu \mathrm{m})$; pontoações radio-vasculares simples, com borda reduzida, horizontal. Fibras de paredes finas e pontoações simples, com aréolas reduzidas em vista radial; septos presentes. Parênquima axial paratraqueal escasso; séries de parênquima axial com 3-4 células. Raios (1-3) 2-3 seriados, com 6-30 células de altura; heterocelulares, com corpo de células procumbentes e margens de células eretas ou quadradas. Células oleíferas ou mucilaginosas, raras, associadas ao parênquima radial.

\section{Nectandra reticulata (Ruiz \& Pav.) Mez}

Anéis de crescimento distintos, marcados por fibras de lenho tardio de paredes espessas. Vasos difusos, solitários e em múltiplos de dois;

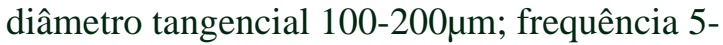
20 por $\mathrm{mm}^{2}$; placas de perfuração simples e escalariformes de poucas barras; pontoações intervasculares alternas, pequenas $(4-7 \mu \mathrm{m})$; pontoações radio-vasculares simples, com borda reduzida, horizontal. Fibras de paredes finas e pontoações simples, com aréolas reduzidas em vista radial; septos presentes. Parênquima axial paratraqueal escasso e vasicêntrico; séries de parênquima axial com 3 células. Raios (1-3) 3 seriados, com 4-35 células de altura; heterocelulares, com corpo de células procumbentes e margens de células eretas ou quadradas. Células oleíferas ou mucilaginosas, associadas ao parênquima axial.

\section{DISCUSSÃO}

Os elementos do tecido xilemático possuem estruturas de notável importância em taxonomia e filogenia. O estudo do xilema mostra algumas características relacionadas às principais tendências de evolução das Angiospermas, notadamente em elementos de vaso (Carlquist, 1962).

A estrutura da madeira em Lauraceae é considerada por Richter (1987) como "uniforme". A combinação convergente de atributos anatômicos caracteriza a família em relação a outras (Stern, 1954; Richter, 1987). Stern (1954) menciona que raios estreitos, geralmente com 1-4 células de largura, parênquima paratraqueal presente em todas as espécies, pontoações intervasculares alternas, células oleíferas/ mucilaginosas na maioria das espécies e porosidade difusa, são características importantes para a identificação de Lauraceae, atributos, estes, observados nas madeiras das espécies aqui tratadas.

Para as madeiras do gênero Nectandra são descritos como mais constantes os seguintes atributos: porosidade difusa; vasos em arranjo diagonal, nunca exclusivamente solitários e sempre acompanhados por múltiplos radiais de poucos elementos; diâmetro tangencial médio (140-200 $\mu \mathrm{m})$; frequência de vasos entre 6-17/ $\mathrm{mm}^{2}$, placas de perfuração exclusivamente simples ou simples e escalariformes; pontoações intervasculares alternas, médias a grandes (9$14 \mu \mathrm{m}$ ); fibras septadas de paredes delgadas a espessas, com pontoações simples, restritas às faces radiais da parede; parênquima axial paratraqueal; raios unisseriados e multisseriados, heterogêneos, geralmente com 2-3 células de largura; e células oleíferas/mucila- 
Tabela 2. Dados quantitativos, médias e respectivos desvios-padrão dos atributos anatômicos e da densidade das madeiras em estudo. Letras diferentes na mesma linha indicam diferenças estatisticamente significativas, com $\mathrm{p}<0,05$.

\begin{tabular}{|c|c|c|c|c|c|c|}
\hline $\begin{array}{l}\text { Atributos da } \\
\text { madeira }\end{array}$ & $\begin{array}{l}\text { Nectandra } \\
\text { lanceolata }\end{array}$ & $\begin{array}{c}\text { Nectandra } \\
\text { Membranacea }\end{array}$ & $\begin{array}{c}\text { Nectandra } \\
\text { oppositifolia }\end{array}$ & $\begin{array}{c}\text { Nectandra } \\
\text { psammophila }\end{array}$ & $\begin{array}{c}\text { Nectandra } \\
\text { puberula }\end{array}$ & $\begin{array}{c}\text { Nectandra } \\
\text { reticulata }\end{array}$ \\
\hline $\begin{array}{l}\text { Altura raios } \\
(\mu \mathrm{m})\end{array}$ & $\begin{array}{c}374.59 \\
( \pm 99.08) \\
b\end{array}$ & $\begin{array}{c}379.52 \\
( \pm 93.41) \\
b\end{array}$ & $\begin{array}{c}384.15 \\
( \pm 78.55) \\
b\end{array}$ & $\begin{array}{c}383.91 \\
( \pm 128.31) \\
b\end{array}$ & $\begin{array}{c}287.32 \\
( \pm 61.43) \\
a\end{array}$ & $\begin{array}{c}356.54 \\
( \pm 65.73) \\
b\end{array}$ \\
\hline $\begin{array}{l}\text { Altura raios } \\
\text { (nº células) }\end{array}$ & $\begin{array}{c}26.47 \\
( \pm 15.25) \\
b\end{array}$ & $\begin{array}{c}23.60 \\
( \pm 8.24) \\
\text { ab }\end{array}$ & $\begin{array}{c}20.07 \\
( \pm 7.67) \\
a b\end{array}$ & $\begin{array}{c}24.27 \\
( \pm 14.66) \\
b\end{array}$ & $\begin{array}{c}15.97 \\
( \pm 6.05) \\
a\end{array}$ & $\begin{array}{c}21.90 \\
( \pm 9.36) \\
\mathrm{ab}\end{array}$ \\
\hline $\begin{array}{l}\text { Comprimento } \\
\text { de elementos } \\
\text { de vaso }(\mu \mathrm{m})\end{array}$ & $\begin{array}{c}471.67 \\
( \pm 97.71) \\
a b\end{array}$ & $\begin{array}{c}505.58 \\
( \pm 113.82) \\
\text { ac }\end{array}$ & $\begin{array}{c}552.46 \\
( \pm 103.25) \\
b c\end{array}$ & $\begin{array}{c}459.45 \\
( \pm 59.99) \\
a\end{array}$ & $\begin{array}{c}524.00 \\
( \pm 140.41) \\
\text { ac }\end{array}$ & $\begin{array}{c}572.91 \\
( \pm 163.46) \\
c\end{array}$ \\
\hline $\begin{array}{l}\text { Comprimento } \\
\text { de fibras } \\
(\mu \mathrm{m})\end{array}$ & $\begin{array}{c}771.20 \\
( \pm 123.49) \\
a\end{array}$ & $\begin{array}{c}1242.25 \\
( \pm 194.55) \\
c\end{array}$ & $\begin{array}{c}1184.51 \\
( \pm 142.04) \\
c\end{array}$ & $\begin{array}{c}990.85 \\
( \pm 150.28) \\
b\end{array}$ & $\begin{array}{c}984.42 \\
( \pm 126.32) \\
b\end{array}$ & $\begin{array}{c}1165.12 \\
( \pm 152.55) \\
c\end{array}$ \\
\hline $\begin{array}{l}\text { Diâmetro } \\
\text { tangencial de } \\
\text { vasos }(\mu \mathrm{m})\end{array}$ & $\begin{array}{c}119.61 \\
( \pm 21.74) \\
a\end{array}$ & $\begin{array}{c}209.50 \\
( \pm 124.99) \\
b\end{array}$ & $\begin{array}{c}127.23 \\
( \pm 20.58) \\
a\end{array}$ & $\begin{array}{c}98.25 \\
( \pm 9.53) \\
a\end{array}$ & $\begin{array}{c}96.36 \\
( \pm 10.31) \\
a\end{array}$ & $\begin{array}{c}106.07 \\
( \pm 11.72) \\
a\end{array}$ \\
\hline $\begin{array}{l}\text { Espessura } \\
\text { parede de } \\
\text { fibras }(\mu \mathrm{m})\end{array}$ & $\begin{array}{c}5.01 \\
( \pm 1.33) \\
b\end{array}$ & $\begin{array}{c}3.93 \\
( \pm 0.79) \\
a\end{array}$ & $\begin{array}{c}6.00 \\
( \pm 1.06) \\
c\end{array}$ & $\begin{array}{c}5.63 \\
( \pm 0.94) \\
\text { bc }\end{array}$ & $\begin{array}{c}5.37 \\
( \pm 0.92) \\
\text { bc }\end{array}$ & $\begin{array}{c}4.89 \\
( \pm 0.90) \\
b\end{array}$ \\
\hline $\begin{array}{l}\text { Frequência d e } \\
\text { vasos } \\
\left(\mathrm{mm}^{2}\right)\end{array}$ & $\begin{array}{c}20.30 \\
( \pm 3.503) \\
c\end{array}$ & $\begin{array}{c}13.87 \\
( \pm 2.94) \\
b\end{array}$ & $\begin{array}{c}9.83 \\
( \pm 2.46) \\
a\end{array}$ & $\begin{array}{c}21.33 \\
( \pm 3.38) \\
c\end{array}$ & $\begin{array}{c}24.63 \\
( \pm 4.47) \\
d\end{array}$ & $\begin{array}{c}13.07 \\
( \pm 3.88) \\
b\end{array}$ \\
\hline $\begin{array}{l}\text { Largura } \\
\text { de raios }(\mu \mathrm{m})\end{array}$ & $\begin{array}{c}48.83 \\
( \pm 7.69) \\
c\end{array}$ & $\begin{array}{c}35.82 \\
( \pm 4.90) \\
a b\end{array}$ & $\begin{array}{c}46.56 \\
( \pm 4.94) \\
c\end{array}$ & $\begin{array}{c}39.32 \\
( \pm 5.22) \\
b\end{array}$ & $\begin{array}{c}24.32 \\
( \pm 4.66) \\
a\end{array}$ & $\begin{array}{c}36.30 \\
( \pm 5.09) \\
a b\end{array}$ \\
\hline $\begin{array}{l}\text { Pontoação } \\
\text { intervascular } \\
(\mu \mathrm{m})\end{array}$ & $\begin{array}{c}5.49 \\
( \pm 1.23) \\
a\end{array}$ & $\begin{array}{c}10.32 \\
( \pm 1.74) \\
c\end{array}$ & $\begin{array}{c}7.63 \\
( \pm 1.29) \\
b\end{array}$ & $\begin{array}{c}5.16 \\
( \pm 0.95) \\
a\end{array}$ & $\begin{array}{c}5.31 \\
( \pm 0.69) \\
a\end{array}$ & $\begin{array}{c}6.92 \\
( \pm 1.05) \\
b\end{array}$ \\
\hline $\begin{array}{l}\text { Frequência d e } \\
\text { vasos }\left(\mathrm{mm}^{2}\right)\end{array}$ & $\begin{array}{c}20.30 \\
( \pm 3.50) \\
c\end{array}$ & $\begin{array}{c}13.86 \\
( \pm 2.94) \\
b\end{array}$ & $\begin{array}{c}9.83 \\
( \pm 2.46) \\
a\end{array}$ & $\begin{array}{c}21.33 \\
( \pm 3.37) \\
c\end{array}$ & $\begin{array}{c}24.63 \\
( \pm 4.47) \\
d\end{array}$ & $\begin{array}{c}13.06 \\
( \pm 3.87) \\
b\end{array}$ \\
\hline $\begin{array}{l}\text { Densidade bá - } \\
\text { sica da madei - } \\
\mathrm{ra}\left(\mathrm{g} / \mathrm{cm}^{2}\right)\end{array}$ & $\begin{array}{c}0.44 \\
\mathrm{a}\end{array}$ & $\begin{array}{c}0.46 \\
\mathrm{c}\end{array}$ & $\begin{array}{c}0.59 \\
\mathrm{f}\end{array}$ & $\begin{array}{c}0.45 \\
\mathrm{~b}\end{array}$ & $\begin{array}{c}0.57 \\
\mathrm{e}\end{array}$ & $\begin{array}{c}0.52 \\
\mathrm{~d}\end{array}$ \\
\hline
\end{tabular}


ginosas frequentemente presentes (Record \& Hess, 1942; Record \& Mell, 1924; Richter, 1987).

Os anéis de crescimento, quando presentes, podem ser usados para fins taxonômicos, apesar dos escassos registros disponíveis, bem como sobre a variabilidade na largura dos mesmos (Carlquist, 1962). As camadas de crescimento, bem definidas nas espécies em estudo, sugerem uma resposta das plantas a condições adversas do ambiente, tais como baixa disponibilidade de água. Tal característica aproxima as espécies do padrão observado na família Lauraceae. Kira (1983) menciona que uma das condições ambientais para a marcação de camadas de crescimento em árvores de regiões tropicais é a ocorrência de precipitação inferior a $60 \mathrm{~mm}$, num período de 60 dias. No município de Matozinhos (MG), a estação seca estende-se de abril a setembro, com precipitação no período entre 6 e $40 \mathrm{~mm}$, o que pode provocar a formação de camadas de crescimento no lenho de $N$. lanceolata e $N$. psammophila. Nos demais municípios de coleta (Araquari, Guaramirim e São Francisco do Sul), o período seco ocorre entre os meses de junho a agosto, com precipitação abaixo de $100 \mathrm{~mm}$ (Climate-Data, 2016).

Alguns atributos associados a elementos de vaso, como tipo de porosidade e arranjo dos mesmos, exibem importância diagnóstica e podem ser usados na indicação de compatibilidade entre táxons (Metcalfe \& Chalk, 1983). A porosidade difusa, registrada nas espécies de Nectandra, é considerada frequente em espécies lenhosas da flora do Brasil (Alves \& Angyalossy-Alfonso, 2000). O mesmo padrão difuso de arranjo de vasos também foi descrito por Oliveira et al. (2001) para N. puberula coletada em Floresta Ombrófila Densa Montana.

$\mathrm{Na}$ maioria das espécies estudadas, observam-se elementos de vaso solitários e em múltiplos radiais. Nectandra membranacea, $N$. oppositifolia, $N$. psammophila e $N$. reticulata apresentaram vasos solitários, seguido por múltiplos de dois, ao passo que em $N$. lanceolata e $N$. puberula a maioria dos mesmos encontra-se em múltiplos de 2-3, seguido por solitários. Metcalfe \& Chalk (1983) consideram não especializada a ocorrência exclusiva de elementos de vaso solitários.

Os elementos de vaso de $N$. psammophila e $N$. puberula apresentam diâmetro tangencial com valores entre 74-118 $\mu \mathrm{m}$; de acordo com Coradin \& Muniz (1991), tais diâmetros são considerados pequenos. Em $N$. lanceolata, o diâmetro tangencial médio de elementos de vaso é de 119,61 $\mu \mathrm{m}$, aproximando-se, bastante, dos valores referidos por Tortorelli (1956). De acordo com Carlquist (2001), vasos de diâmetros tangenciais maiores (médio, 100-200 $\mu \mathrm{m}$, e grande, $=200 \mu \mathrm{m}$, segundo IAWA, 1989) e forma circular favorecem o transporte individual de grandes volumes de água por unidade de tempo. Desta forma, sugere-se que Nectandra membranacea $(=200 \mu \mathrm{m})$, espécie com vasos de diâmetro grande, bem como $N$. lanceolata, $N$. oppositifolia e $N$. reticulata, com diâmetros tangenciais médios (100-200 $\mu \mathrm{m})$, são especializadas em lidar com grandes volumes de água, comparadas a $N$. psammophila e $N$. puberula, as quais, segundo IAWA (1989), possuem vasos pequenos $(50-100 \mu \mathrm{m})$. PolancoTapia \& Grande-Pulido (2009) referem que vasos pequenos oferecem maior resistência à cavitação e menor propensão a embolismos por estresse hídrico, resultando em maior segurança para as colunas d'água (Woodcock, 1994).

Para Carlquist (1962), o número de vasos por $\mathrm{mm}^{2}$ é característica taxonômica importante, sendo muito numeroso em espécies de Lauraceae (IPT, 1989), o qual contrasta com os resultados deste trabalho. As espécies coletadas em áreas de menor precipitação (FES), como Nectandra lanceolata e N. psammophila, apresentaram maior frequência de vasos. $N$. membranacea e $N$. reticulata, provenientes de áreas de alta precipitação (FODTB), bem como $N$. oppositifolia (de Restinga), apresentaram baixas frequências de vasos. Segundo Metcalfe \& Chalk (1985), esta característica é considerada plástica e, em função do ambiente, pode variar dentro de uma mesma espécie. Logo, suge- 
re-se que a alta densidade de fibras (Tabela 2) colabora para que os vasos mantenham a grande condução de água nestas espécies.

Quanto às placas de perfuração, em $N$. membranacea registrou-se a combinação de placas simples com raras foraminadas. $\mathrm{O}$ tipo de placa de perfuração, segundo Silva \& Medeiros (2000), é característica anatômica relevante na distinção entre gêneros de Lauraceae. León (2000) e Leon \& Espinoza de Pernía (2001) utilizaram este caráter para separar, em grupos, 17 espécies de Ocotea e 18 espécies de Nectandra. No presente estudo, todas as espécies de Nectandra apresentaram placas de perfuração simples e escalariformes. Entretanto, $N$. membranacea apresentou placas de perfuração simples e raras foraminadas. Para $N$. lanceolata, Tortorelli (1956) cita apenas placas de perfuração simples; os resultados da presente pesquisa, no entanto, comprova a ocorrência do tipo escalariforme com poucas barras, embora pouco frequente, concordando, neste aspecto, com Hernandez (1985).

Pontoações intervasculares alternas médias a grandes (9-14 $\mu \mathrm{m})$ são descritas como mais constantes para o gênero Nectandra (Richter, 1987). Segundo IAWA (1989), as espécies aqui estudadas apresentam pontoações intervasculares alternas pequenas $(4-7 \mu \mathrm{m})$, com exceção de $N$. membranacea, que apresentou pontoações intervasculares grandes $(=10 \mu \mathrm{m})$. Carlquist (1975) cita que a estrutura anatômica do lenho é determinada por fatores genéticos, mas alterações quantitativas podem ocorrer em resultado de efeito ambiental, seja climático, edáfico ou fisiográfico, levando o xilema a adaptações anatômicas mais favoráveis em relação às condições do habitat.

Richter (1987) ressalta a importância da associação entre a forma e o tamanho das pontoações radio-vasculares como importante na identificação taxonômica das Lauraceae. No presente trabalho observaram-se pontoações radio-vasculares simples com borda reduzida, horizontal.
Dentro da família Lauraceae predominam fibras libriformes e septadas, com pontoações simples (Metcalfe, 1987). Carlquist (1962) reconhece que a presença de septos em fibras é caráter útil na comparação de madeiras. No entanto, Fahn (1990) sugere que tal estrutura pode ser interpretada como suporte mecânico, pois as fibras septadas desenvolvem delgadas paredes transversais (septos) através do lume da célula, posteriormente à formação das paredes secundárias (Dickison, 2000).

Reconhecido como um dos mais importantes parâmetros na identificação anatômica do lenho, o parênquima axial constitui a entrada das chaves clássicas de identificação (Chimelo et al., 1993). Richter (1987) destaca o tipo e a distribuição do parênquima axial como característica anatômica de grande importância na identificação de Lauraceae. Esta característica também foi importante na segregação de algumas espécies de Ocotea e Nectandra estudadas por Czarneski et al. (2001) e Oliveira et al. (2001). No presente trabalho, as espécies estudadas apresentaram parênquima paratraqueal escasso; segundo Van Vliet et al. (1981), é para compensar o escasso tecido de armazenamento que se desenvolvem fibras septadas, as quais funcionam como séries de parênquima.

No tocante à estrutura radial, em todas as espécies estudadas observam-se raios multisseriados, De acordo com Carlquist (1966), raios multisseriados predominam em espécies de ambientes secos, ao passo que em amostras de floresta os raios são mais estreitos.

A presença de células oleíferas/mucilaginosas é comumente referida para a maioria das espécies de Lauraceae (Richter, 1987). Este atributo foi considerado importante por Leon (2000), León \& Espinoza de Pernía (2001) e Oliveira et al. (2001) na identificação de algumas espécies dos gêneros Ocotea e Nectandra. Dentre as espécies estudadas neste trabalho, Nectandra lanceolata, N. membranacea e $N$. oppositifolia não apresentaram células oleíferas/ mucilaginosas. 

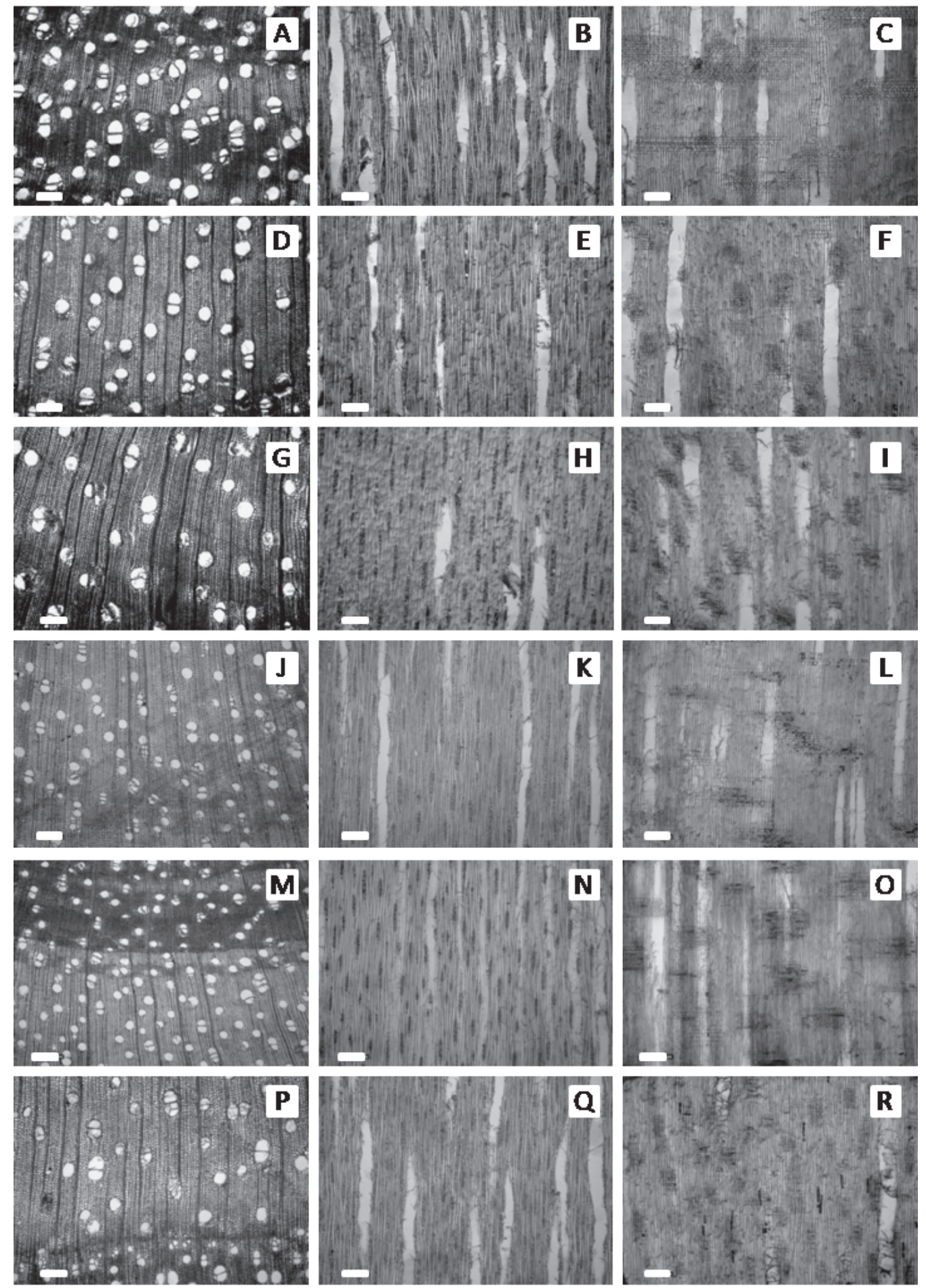

FIGURA 1 - Fotomicrografias das madeiras estudadas. A-C, Nectandra lanceolata. D-F, Nectandra membranacea. G-I, Nectandra oppositifolia. J-L, Nectandra psammophila. M-O, Nectandra puberula. P-R, Nectandra reticulata. Seções transversal, longitudinal tangencial e longitudinal radial são mostradas da esquerda para a direita. Barra de escala $=200 \mu \mathrm{m}$. 
A análise comparativa das seis espécies de Nectandra reforça a grande uniformidade estrutural do gênero. Contudo, diferenças observadas na anatomia da madeira podem contribuir para a segregação das espécies, salientando-se: tipo de placa de perfuração, presença de tilos, diâmetro de pontoações intervasculares, comprimento de fibras, altura de raios, bem como presença de raios agregados e de células oleíferas/mucilaginosas. Ressalta-se que diferenças nos atributos quantitativos entre as espécies podem ser respostas plásticas às condições ambientais, isto é, peculiares aos ambientes de desenvolvimento das mesmas.

\section{REFERÊNCIAS BIBLIOGRÁFICAS}

ALLEN, C.K. Notes on Lauraceae of tropical America I. The generic status of Nectandra, Ocotea and Pleurothyrium. Phytologia, n. 13, p. 221-233, 1966.

ALVES, E.S.; ANGYALOSSY-ALFONSO, V. Ecological trends in the Wood anatomy of some Brazilian species: 1. Growth rings and vessels. IAWA Journal, Utrecht, v. 21, n. 1, p. 3-30, 2000.

APG III. An update of the Angiosperm Phylogeny Group classification for the orders and families of flowering plants: APG III. Botanical Journal of the Linnean Society, n. 161, p. 105-121, 2009.

ABNT - Associação Brasileira de Normas Técnicas. Comissão de estudo de madeira para fabricação de pasta celulósica. NBR 11941. Rio de Janeiro, 2002.

BAAS, P.; WHEELER E.; CHASE M. Dicotyledonous wood anatomy and the APG system of Angiosperm classification. Bot. J. Linn. Soc., n. 134 , p. 3-17, 2000.

BAITELLO, J.B.; HERNÁNDES, F.G.L.; MORAES, P.L.R. de; ESTEVES, R.; MARCOVINO, J.R. Lauraceae. In: WANDERLEI, M.G.L.; SHEPHERD, G.J.;GIULIETTI, A.M.; MELHEM, T.S. Flora fanerogâmica do Estado de São Paulo. São Paulo: Editora Rima, 2003. v.3. p.149-223.

BARRETO, R.C. O gênero Nectandra Rol. ex Rottb. (Lauraceae) no Estado de Pernambuco. Acta Botânica Brasilica, v. 1, n. 2, p. 63-71, 1988.

BARRETO, R.C. O gênero Ocotea Aubl. (Lauraceae) no Estado de Pernambuco. Anais do $36^{\circ}$ Congresso Nacional de Botânica, 1, 1990. p. 175199.
BARROSO, G.M. et al. Sistemática de Angiospermas do Brasil. Viçosa: Universidade Federal de Viçosa, 2002. v. 1.

BERLYN, G.P.; MIKSCHE, J.P. Botanical microtechnique and cytochemistry. Ames: The Iowa State University Press, 1976.

BERNARDI, L. Lauráceas. Mérida: Talleres Graficos Universitários, 1962.

BORCARD, D.; GILLET, F.; LEGENDRE, P. Numerical Ecology with R. Ed. Springer, 2011.

CARLQUIST, S. Comparative plant anatomy: A guide to taxonomic and evolucionary application of anatomical data in Angiosperms. New York: Holt, Rinehart and Winston, 1962. 145 p.

CARLQUIST, S. Ecological strategies of xylem evolution. Berkeley/Los Angeles: University of California Press, 1975. 259p.

CARLQUIST, S. Comparative Wood Anatomy: Systematic, Ecological and Evolutionary aspects of Dicotyledon Woods. New York: Springer Verlag, 2001.

CARVALHO, P.E.R. Canela-Branca. Embrapa Floresta, Colombo, PR, 2002. ISSN 1517-5278.

CASTIGLIONI, J.A. El leño secundario de las especies argentinas de Nectandra. Revista de Investigaciones Forestales, v. 3, n. 1, p. 1-15, 1962.

CHANDERBALI, A.S.; VAN DER WERFF, H.; RENNER, S.S. Phylogeny and historical biogeography of Lauraceae: evidence from the chloroplast and nuclear genomes. Annals of the Missouri Botanical Garden, n. 88, p. 104-134, 2001.

CHIMELO, J.P.; ZENID, G.J; MIRANDA, M.J.A.C.; CECCANTINI, G.C.T. IMAC: Identificação de madeiras brasileiras com auxílio do computador. São Paulo: IPT, 1993. 56p.

CLIMATE-DATA.ORG. 2016. Dados climáticos para cidades mundiais. Publicação eletrônica: http:// en.climate-data.org/.

COE-TEIXEIRA, B. Lauraceae do gênero Ocotea, do Estado de São Paulo. Rodriguesia, v. 52, p. 55-190, 1980.

CORADIN, T.R.V.; MUÑIZ, G.L.B. Normas de procedimentos em estudos de anatomia de madei$r a$ : I. Angiospermae; II. Gimnospermae. Brasília: Comissão de estudos CE 11.01.07:001 ABNT, 1991. $19 \mathrm{p}$.

CRAWLEY, M.J. Statistical computing: an introduction to data analysis usingsplus. London: John Wiley \& Sons, 2007. 942p.

CRONQUIST, A. The Evolution and Classification of Flowering Plants. New York: The New York Botanical Garden, 1988. 
CZARNESKI, C.M.; FREITAS, G.F.; CORADIN, V.T.R.; CAMARGOS, J.A.A. Estudo anatômico comparativo do lenho de quatro espécies da família Lauraceae ocorrentes na Amazônia. Brasil Florestal, n. 70, p. 48-60, 2001.

DICKISON, W.C. Interactive plant anatomy. Harcout Academic Press, 2000. 532 p.

ESAU, K. Plant anatomy. New York: John Wiley \& Sons Inc., 1965. 767 p.

FAHN, A. Plant Anatomy. New York: Pergamon Press, 1990. 558 p.

GENTRY, A. Changes in plant community diversity and floristic composition on environmental and geographical gradients. Annals of the Missouri Botanical Garden, n. 69, p. 557-593, 1988.

HEO, K.; VAN DER WERFF, H.; TOBE, H. Embriology and relationships of Lauraceae (Laurales). Botanical Journal of the Linnean Society, v. 126, p. 295-322, 1998.

HERNANDEZ, H. Estudio del xilema secundario de las Lauraceae argentinas. Lilloa, v. 2, n. 38, p. 41-71, 1985.

IAWA. IAWA list of microscopic features for hardwood identification. IAWA Bulletin, v. 10, n. 3, p. 219-332, 1989.

IPT. Fichas das características de madeiras brasileiras. Instituto de PesquisasTecnológicas - IPT, São Paulo, 1989.

IUCN. 2009. IUCN Red list of threatened species. Disponível em: http://www.biodiversitas.org.br/ floraBr/iucn.pdf.

JOHANSEN, D.A. Plant Microtechnique. New York: McGraw-Hill Book Company, 1940. 523 p.

JUDD, W.S.; CAMPBELL, C.S.; KELLOGG, E.A.; STEVENS, P.F. Plant Systematics, a phylogenetic approach. Massachusetts: Sinauer Associates, Inc. Publischers, 1999. 464 p.

KIRA, T. Ecology of tropical Forest. Tokyo: J. Shin., 1983. $251 \mathrm{p}$.

KOSTERMANS, A.J.G.H. A historical survey of Lauraceae. Journal for Scientific Research, n 1, p. 83-95, 113-127, 141-159, 1952.

KOSTERMANS, A.J.G.H. Lauraceae. Reinwardtia, v. 4, n. 2, p. 193-256, 1957.

LEÓN, W.J. Anatomía del leño de 17 especies del género Ocotea Aublet (Lauraceae). Pittieria, v. 1, n. 29, 30, p. 53-65, 2000.

LEÓN, W.J.; Espinoza de Pernía, N. Estudio anatômico del leño de 18 especies del género Nectandra Rol. ex Rottb. (Lauraceae). Revista Forestal Venezolana, v. 45, n. 1, p. 33-44, 2001.

LORENZI, H.; SOUZA, V.C. Botânica Sistemática: guia ilustrado para identificação das famílias de Fanerógamas nativas e exóticas no Brasil, base- ado em APG III. Nova Odessa: Instituto Plantarum, 2012.

MADRIÑÁN, S. Lauraceae: Rhodostemonodaphne. Flora Neotropica Monograph, 92: 1-102, 2004.

MARCATI, C.R.; OLIVEIRA, J.S.; MACHADO, S.R. Growthrings in cerrado Wood species: occurrence and anatomical markers. Biota Neotropica, Campinas, v. 6, n. 3, p.1-31, 2006.

MELO JUNIOR, J.C.F.; AMORIM, M.W.; SILVEIRA, E.R. 2014. A xiloteca (coleção Joinvillea - JOIw) da Universidade da Região de Joinville. Rodriguesia, v. 65, p. 1.057 - 1.060.

METCALFE, C.R. 1987. Anatomy of the Dicotyledons Magnoliales, Illiciales, and Laurales. 2: ed. Clarendon Press, Oxford. v. 3. 224 p.

METCALFE, C.R.; CHALK L. Anatomy of the Dicotyledons. Oxford/London: Clarendon Press, 1957. v. 1. p. 669-674.

METCALFE, C.R.; CHALK L. Anatomy of the Dicotyledons. Oxford/London: Clarendon Press, 1979.

METCALFE, C.R.; CHALK L. Anatomy of the Dicotyledons Wood structure and conclusion of the general introduction. Oxford: Clarendon Press, 1983. v. 2. 297 p.

OLIVEIRA, W.C.; CALLADO, C.H.; MARQUETE, O. Anatomia do lenho de espécies do gênero Nectandra Rol. ex Rottb. (Lauraceae). Rodriguesia, Rio de Janeiro, v. 52, n. 81, p. 125134, 2001.

OCCHIONI, P.; FILHO A.M. Estudo anatômico do lenho secundário do Puchury-Rana, Ocotea fragrantissima Ducke. Rodriguésia, Rio de Janeiro, n. 21, p. 1-12, 1947.

PAIVA, J.G.A.; FANK-DE-CARVALHO, S.M.; MAGALHÃES, M.P.; GRACIANO-RIBEIRO, D. Verniz vitral incolor 500: uma alternativa de meio de montagem economicamente viável. Acta Botanica Brasilica, v. 20, n. 2. p. 257-264, 2006.

PEDRALLI, G. O gênero Ocotea Aublet (Lauraceae) no Estado do Rio Grande do Sul, Brasil. In: XXXVII Congresso Nacional de Botânica. Universidade Federal de Ouro Preto/Minas Gerais. Brasil, p. 426-466, 1986.

POLANCO-TAPIA, C.; GRANDE-PULIDO, D.C. Análisis ecoanatómico, evolutivo y comparativo de la madera de 40 especies de dos asociaciones del Bosque altoandino colombiano. Revista Colômbia Forestal, n. 12, p. 183203, 2009.

QUINET, A. 2015. Nectandra in Flora do Brasil 2020. Jardim Botânico do Rio de Janeiro. Disponível em: <http://floradobrasil.jbrj.gov.br/ 
reflora/floradobrasil/FB8424>. Acesso em: 02 Abr. 2016.

QUINET, A.; ANDREATA, R. H. P. Lauraceae Jussieu na Reserva Ecológica de Macaé de Cima, município de Nova Friburgo, Rio de Janeiro, Brasil. Rodriguesia, Rio de Janeiro, v. 53, n. 82, p. 59-121, 2002.

RECORD, S.J. 1944. Keys to american woods. Tropical Woods, n. 78, p. 35-45.

RECORD, S.J.; HESS, R.W. American timbers of the family Lauraceae. Tropical Woods, n. 69, p. 7-33, 1942.

RECORD, S.J.; MELL, C.D. Timbers of Tropical America. New Haven: Yale University Press, 1924. $610 \mathrm{p}$.

REFLORA. Disponível em: http://floradobrasil.jbrj.gov.br/. Acesso em 21 Jan. 2016.

RICHTER, H.G. 1987. Mature Secondary Xylem. In: METCALFE, C.R. Anatomy of the Dicotyledons. Oxford: Clarendon Press, 1987. III. p. 162-171.

RIZZINI, C.T. Árvores e madeiras úteis do Brasil. São Paulo: E. Blücher, 1971. 286 p.

ROHWER, J.G. Prodromuseiner monographie der Gattung Ocotea Aubl. (Lauraceae) sensu lato. Hamburg: Hubert \& Co., 1986. 278 p.

ROHWER, J.G. Lauraceae. In: KUBITZKI, K., ROHWER, J.G.; BITTRICH, V. (Eds.). The Families and Genera of Vascular Plants. Berlin: Springer-Verlag, 1993. v. 2. p. 366-391.

ROHWER, J.G. Toward a phylogenetic classification of the Lauraceae: evidence from matK sequences. Systematic Botany, v. 25, n. 1, p. 60$71,2000$.

ROHWER, J.G.; KUBITZKI, K. Ecogeographical differentiation in Nectandra (Lauraceae) and its historical implications. Acta Botanica, n. 106, p. 88-99, 1993.

ROHWER, J.G.; RUDOLPH, B. Jumping genera: the phylogenetic positions of Cassytha, Hypodaphnis and Neocinnamomum (Lauraceae) based on different analyses of trn Kintron sequences. Annals of the Missouri Botanical Garden, n. 92, p. 153-178, 2005.

STERN, W.L. Comparative anatomy of xylem and phylogeny of Lauraceae. Tropical Woods, n. 100, p. 1-75, 1954.

SANTOS, S.O.; ALVES, M. Flora da Usina São José, Igarassu, Pernambuco: Lauraceae. Rodriguésia, Rio de Janeiro, v. 63, n. 3, p. 689-703, 2012.

SASS, J.E. Elements of Botanical Microtechnique. New York, London: McGraw-Hill, 1958. 222 p.

SOUZA, V.C.; LORENZI, H. Botânica Sistemática: guia ilustrado para identificação das famílias de Fanerógamas nativas e exóticas do Brasil, baseado em APG II. Nova Odessa: Editora Plantarum, 2008.

STUESSY, E. Plant taxonomy. The evolution of comparative data. New York: Columbia University Press, 1991. 514 p.

TORTORELLI, L.A. Maderas y bosques argentinos. Buenos Aires: ACME, 1956. 910 p.

VAN DER WERFF, H. A key to the genera of Lauraceae in the New World. Annals of the Missouri Botanical Garden, n. 78, p. 377-387, 1991.

VAN DER WERFF, H.; RICHTER, H. G. Toward an improved classification of Lauraceae. Annals of the Missouri Botanical Garden, n. 83, p. 409418, 1996.

VAN VLIET, G.J.C.M.; KOEK-NOORMAN, J.; WELLE, B.J.H.T. Wood anatomy, classification and phylogeny of the Melastomataceae. Blumea, n. 27, p. 463-473, 1981.

VATTIMO-GIL, I. O gênero Ocotea Aubl. no Nordeste do Brasil. (Lauraceae). Rodriguesia, Rio de Janeiro, v. 23/24, n. 35, p. 241-251, 1960/ 1961.

VATTIMO-GIL, I. Lauraceae do Estado da Guanabara. Rodriguesia, Rio de Janeiro, v. 25, n. 37, p. 75-223, 1966.

WHEELER, E.A.; PEARSON, R.G.; LA PASHA, C.A.; ZACK, T.; HATLEY, W. Computer-aided wood identification - reference manual. Madison: North Carolina Agricultural Research Service, Department of Wood and Paper Science, 1986. $160 \mathrm{p}$.

WOODCOCK, D.W. Occurrence of woods with a gradation in vessel diameter across the ring. IAWA Journal, n. 15, p. 377-385, 1994. 